\title{
openheart Associations between cardiac troponin, mortality and subsequent use of cardiovascular services: differences in sex and ethnicity
}

\author{
David E Winchester, ${ }^{1,2}$ Kristopher Kline ${ }^{3}$ Christopher Estel, ${ }^{1}$ Dhruv Mahtta, ${ }^{3}$ \\ Sean Taasan, ${ }^{4}$ Franck W Peacock ${ }^{5}$
}

\begin{abstract}
- Additional material is published online only. To view please visit the journal online (http://dx.doi.org/10.1136/ openhrt-2017-000713).
\end{abstract}

To cite: Winchester DE, Kline K, Estel C, et al. Associations between cardiac troponin, mortality and subsequent use of cardiovascular services: differences in sex and ethnicity. Open Heart 2018;5: 000713. doi:10.1136/ openhrt-2017-000713

Received 31 August 2017 Revised 5 December 2017 Accepted 18 January 2018

\section{Check for updates}

${ }^{1}$ Division of Cardiovascular Medicine, Department of Medicine, College of Medicine, University of Florida, Gainesville, Florida, USA

${ }^{2}$ Cardiology Section, Medical Service, Malcom Randall VA Medical Center, Gainesville, Florida, USA

${ }^{3}$ Department of Medicine, College of Medicine, University of Florida, Gainesville, Florida, USA

${ }^{4}$ College of Medicine, University of Florida, Gainesville, Florida, USA

${ }^{5}$ Baylor College of Medicine, Houston, Texas, USA

Correspondence to Dr David E Winchester; david. winchester@medicine.ufl.edu

\section{ABSTRACT}

Background The impact of cardiac troponin (cTn) testing on the downstream use of cardiovascular services is not well understood. We conducted this large-scale single centre cohort study to investigate the patterns of testing that result from the use of cTn.

Methods We conducted this investigation using data collected between 1 January 2013 and 18 December 2015 from an academically affiliated tertiary care centre. Data from all hospitalised patients evaluated with cTn (Roche Elecsys cTn-T) assay were collected from our integrated data repository and divided into two cohorts: all cTn assays negative $(<0.03 \mu \mathrm{g} / \mathrm{L})$ versus at least one elevated $(\geq 0.03 \mu \mathrm{g} / \mathrm{L})$. The main outcomes were the frequency of use cardiovascular services and mortality.

Results Among 26663 subjects, $18.6 \%$ had at least one elevated cTn assay; acute myocardial infarction was diagnosed in $3.9 \%$ overall. More men received cardiac catheterisation and cardiology consultation (OR 1.29, $95 \% \mathrm{Cl} 1.20$ to 1.39 and $\mathrm{OR} 1.45,95 \% \mathrm{Cl} 1.31$ to 1.61 ) while African-American patients were less likely to have either catheterisation (OR $0.85,95 \% \mathrm{Cl} 0.77$ to 0.93 ) or consultation (OR $0.72,95 \% \mathrm{Cl} 0.63$ to 0.82 ) performed. Mortality was associated with detectable cTn (HR 2.05, $\mathrm{P}<0.0001$ ).

Conclusions Among hospitalised patients evaluated with cTn, we observed patterns of underuse and overuse of cardiovascular services. These patterns may have further relevance when high-sensitivity cTn assays are available in the USA. Sex and race-based disparities in cardiovascular services persist.

\section{BACKGROUND}

Myocardial ischaemia and acute myocardial infarction (AMI) are common diagnoses with potentially serious morbidity and mortality. Missed AMI is a common reason for medical malpractice lawsuits in the inpatient and outpatient settings. Symptoms of AMI can be protean, ranging from silent to haemodynamic collapse. ${ }^{1}$ Critically ill patients may not be able to report their symptoms, potentially perplexing physicians if their clinical status suddenly deteriorates. Despite documents
Key messages

What is already known about this subject? Physicians are aware that a substantial proportion of hospitalised patients are evaluated with cardiac troponins and other cardiovascular tests; the scope and magnitude of such testing is not well documented.

What does this study add?

This study demonstrates that contemporary use of cardiac troponin results in a low diagnostic yield for acute myocardial infarction. Elevation in cardiac troponin is associated with substantial demand for cardiovascular services as well as sex and ethnicitybased disparities in care.

How might this impact on clinical practice? Health systems should work to minimise disparities in cardiovascular care. Indiscriminate use of cardiac troponin and downstream testing may be a focus for reducing low-value care. Adoption of higher sensitivity troponin assays may strain available cardiac testing resources

such as the universal definition of MI, which guides clinicians towards a common understanding about myocardial pathology and encourage judicious use of cardiac troponin (cTn), concern about missed diagnoses contributes to the use of cTn to assess for myocardial damage in a wide array of patients. $^{2}$

Investigators have evaluated the test performance characteristics of cTn for decades. In general, cTn is a highly sensitive marker of myocardial damage with poor specificity for AMI. Because the positive and negative predictive values of a given test are dependent on the prevalence of the condition being tested for, the diagnostic value of a given test is degraded if used indiscriminately. ${ }^{3}$ While cTn is a quick and easy test to order, subsequent resource use testing such as echocardiography, nuclear myocardial perfusion 
imaging, cardiac catheterisation and cardiology consultation are associated with non-trivial risks and costs.

The impact of using cTn in minimally selected populations, which may then drive demand for cardiovascular services, has not been well studied. Cardiovascular service demand may dramatically change with the approval of high-sensitivity cTn (hscTn) assays for use in the USA. We conducted a large-scale cohort study to investigate the association between cardiovascular services use and the real-world use of cTn assays. We hypothesised that patients with elevated cTn levels would undergo more cardiovascular testing compared with patients without elevation in cTn.

\section{METHODS}

We conducted a retrospective cohort study using data gathered from a single, academic, tertiary care centre. Subjects were identified using our integrated data repository based on the search characteristics that they were 18 years of age or older, inpatient status and had at least one cTn assay performed during their hospitalisation between 1 January 2013 and 18 December 2015. The cTn assay used at our facility is the Elecsys cTn-T assay (Roche Diagnostics, Indianapolis, Indiana, USA). Our institution defines $0.03 \mu \mathrm{g} / \mathrm{L}$ (99th percentile) as the cutpoint for abnormal. We defined two cohorts: (1) any cTn value greater than or equal to the $0.03 \mu \mathrm{g} / \mathrm{L}$ threshold and (2) all values normal. For feasibility of the statistical analysis, only the first five sample values were considered. Our institutional review board reviewed and approved the research protocol with a waiver for obtaining informed consent. Roche Diagnostics funded the study through an investigator-initiated funding request. Roche had no role in the study design or conduct, data analysis or drafting of the manuscript.

We gathered the data elements from the electronic health record using automated data extraction. Baseline characteristics included age, sex, African American race (self-reported), chronic kidney disease (CKD), coronary artery disease (CAD), hypertension (HTN), congestive heart failure (CHF), atrial fibrillation/flutter, current tobacco use, stroke/transient ischaemic attack and chronic obstructive pulmonary disease. Data on body mass index were not reliably recorded and therefore not used for this investigation. Prior medical diagnoses were taken from the 'problem list' input by clinicians, no secondary definitions were applied. The Charlson Comorbidity Index is automatically calculated by the data repository software; we used the Charlson score as a binary variable ( $\leq 4 \mathrm{vs}>4) .{ }^{45}$ Subsequent hospital diagnoses were identified based on International Classification of Disease- 9 codes provided in online supplementary table S1: AMI, chronic CAD, arrhythmia including atrial or ventricular, CHF, takotsubo cardiomyopathy, sepsis, CKD, acute kidney injury, haemorrhagic stroke, thrombotic stroke, aortic dissection and acute pulmonary embolus. Cardiovascular procedures performed during hospitalisation were identified using Current Procedural Terminology codes and are provided in online supplementary table S1, and include cardiac MRI, cardiac CT, other chest CT, single photon emission CT (SPECT), ventilation/perfusion scan, cardiopulmonary resuscitation, cardioversion, ECG, exercise treadmill test, transthoracic echocardiogram (TTE), transoesophageal echocardiogram, cardiac catheterisation, coronary bypass surgery and percutaneous coronary intervention. We determined from billing data if cardiology consultation was performed for each subject. Vital status from the Social Security Death Index is incorporated into our integrated data repository. We conducted our search for mortality data greater than 6 months after the final hospitalisation date.

The main outcomes were the frequency of use of ECG, non-invasive imaging, cardiac catheterisation and cardiology consultation between the cohorts. Binary associations were calculated using Mantel-Haenszel $\mathrm{X}^{2}$ test. We performed a secondary manual chart review on a $5 \%$ sample of patients who did not have a documented ECG in our administrative to confirm the prevalence of cardiovascular service use. As secondary outcomes, we used the log-rank test and constructed a model of Cox proportional HR for mortality; logistic regression models were used to determine independent associations with cardiology consultation and cardiac catheterisation. Variables eligible for entry to the Cox proportional hazard model for mortality included all baseline medical condition, new diagnoses during hospitalisation, age (by year), African-American race, female sex and elevation of troponin. Kaplan-Meier plots were created to display the risk of mortality related to elevated troponin. ORs were calculated with logistic regression models; variables included in the models of catheterisation and consultation were all baseline medical conditions, age (by year), African-American race, female sex and elevation of troponin. Power calculations were not performed a priori for this population-based investigation. All statistical analyses were performed two-sided, using SAS V.9.4 (Cary, North Carolina, USA) The investigation and manuscript were conducted in accordance with the STROBE guidelines for observational studies. ${ }^{6}$

\section{RESULTS}

\section{Baseline characteristics}

Data on the 26663 subjects are described in table 1 . A total of $4962(18.6 \%)$ patients had a cTn elevation. African-American race was not different between the groups (22.0\% for both cohorts). Male sex was prevalent in $50.1 \%$ of the population and more common in the elevated cTn cohort $(58.0 \%$ vs $48.3 \%, \mathrm{P}<0.0001)$. Rates of medical history were as follows: HTN $(19.5 \%)$, diabetes mellitus (9.8\%), CAD (6.3\%) and CHF (2.9\%). Only $3.93 \%$ of patients with a cTn ordered were subsequently diagnosed with myocardial infarction. 
Table 1 Baseline characteristics and medical history for $n=26663$ subjects

\begin{tabular}{|c|c|c|c|c|c|c|c|}
\hline \multirow[b]{2}{*}{ Age (years) } & \multicolumn{2}{|c|}{$\begin{array}{l}\text { Troponin }<0.03 \\
\mathrm{n}=21701\end{array}$} & \multicolumn{2}{|c|}{$\begin{array}{l}\text { Troponin } \geq 0.03 \\
\mathrm{n}=4962\end{array}$} & \multirow{2}{*}{$\begin{array}{l}\mathbf{P} \\
<0.0001\end{array}$} & \multirow[t]{2}{*}{ OR } & \multirow[t]{2}{*}{$95 \% \mathrm{Cl}$} \\
\hline & 60.2 & 16.2 & 64.9 & 15.4 & & & \\
\hline African-American & 4745 & $22.0 \%$ & 1076 & $22.0 \%$ & 0.95 & 0.96 & 0.93 to 1.08 \\
\hline Male & 10481 & $48.3 \%$ & 2879 & $58.0 \%$ & $<0.0001$ & 1.48 & 1.39 to 1.57 \\
\hline Diabetes mellitus & 1976 & $9.1 \%$ & 624 & $12.6 \%$ & $<0.0001$ & 1.44 & 1.30 to 1.58 \\
\hline Chronic kidney disease & 583 & $2.7 \%$ & 516 & $10.4 \%$ & $<0.0001$ & 4.20 & 3.71 to 4.75 \\
\hline Coronary artery disease & 1207 & $5.6 \%$ & 486 & $9.8 \%$ & $<0.0001$ & 1.84 & 1.65 to 2.06 \\
\hline Hypertension & 4175 & $19.2 \%$ & 1011 & $20.4 \%$ & 0.07 & 1.07 & 1.00 to 1.16 \\
\hline Congestive heart failure & 520 & $2.4 \%$ & 266 & $5.4 \%$ & $<0.0001$ & 2.31 & 1.98 to 2.68 \\
\hline Atrial fibrillation or flutter & 992 & $4.6 \%$ & 339 & $6.8 \%$ & $<0.0001$ & 1.53 & 1.35 to 1.74 \\
\hline Current tobacco use & 775 & $3.6 \%$ & 125 & $2.5 \%$ & 0.0002 & 0.70 & 0.58 to 0.85 \\
\hline Stroke or transient ischaemic attack & 1546 & $7.1 \%$ & 382 & $7.7 \%$ & 0.16 & 1.09 & 0.97 to 1.22 \\
\hline Chronic obstructive pulmonary disease & 1148 & $5.3 \%$ & 255 & $5.1 \%$ & 0.67 & 0.97 & 0.84 to 1.12 \\
\hline Charlson Comorbidity Index $>4$ & 1990 & $9.2 \%$ & 717 & $14.5 \%$ & $<0.0001$ & 1.67 & 1.53 to 1.83 \\
\hline
\end{tabular}

\section{CARDIOVASCULAR SERVICES}

The most common test performed in our study population was an ECG. In our administrative data, ECG was documented for $75.6 \%$ of subjects. When we performed a manual review of charts for patients without a documented ECG, we found the correct estimate to be $4.9 \%$. Undercounting in administrative data was due to ECGs being performed in the prehospital setting or by unit staff without an official order. When ECG was truly not performed, it was typically because of another evident diagnosis (eg, stroke, pulmonary embolus or trauma). TTE was the second most common test, performed in $57.6 \%$ of all patients $(79.4 \%$ for elevated cTn vs $52.6 \%, \mathrm{P}<0.0001$ ) (table 2). Cardiology consultation was performed in the minority of both cohorts $(13.8 \%$ overall; $29.6 \%$ for the elevated cTn cohort vs $10.2 \%$ for the not elevated $c$ Tn cohort, $\mathrm{P}<0.0001$ ). If the $c$ Tn was elevated, invasive coronary angiography and revascularisation procedures were more common, with non-invasive diagnostics occurring less often (table 2). In the secondary chart review, the use of cardiology consultation, nuclear stress testing and TTE were not different from the observations in the administrative data.

\section{HOSPITAL DIAGNOSES}

AMI was an uncommon diagnosis $(3.9 \%$ overall $)$ and was more frequent in the elevated cTn cohort $(20.0 \%$ vs $0.3 \%, \mathrm{P}<0.0001$ ) (table 2 ). The sensitivity, specificity, positive predictive value and negative predictive values for a diagnosis of AMI, based on the elevation of at least one cTn assay, were $94.8 \%, 84.5 \%, 20.0 \%$ and $99.7 \%$, respectively. Chronic cardiovascular disease, CHF, arrhythmia, and both acute and chronic kidney disease were common in both elevated and non-elevated cTn cohorts with higher likelihood of each seen in the elevated cTn cohort. Takotsubo cardiomyopathy was seen in $2.0 \%$ of those with elevated cTn.

\section{MORTALITY}

Overall, 4697 patients died during the study period. In unadjusted comparison, subjects with elevated cTn had more than twice the likelihood of mortality compared with those with no cTn elevation $(35.9 \%$ vs $13.8 \%$, OR $3.49,95 \%$ CI 3.25 to $3.74, \mathrm{P}<0.0001)$. In our Cox proportional hazard model, several baseline characteristics and hospital diagnoses were associated with mortality (table 3). Elevated Charlson Comorbidity Index (HR 3.328, $\mathrm{P}<0.0001)$ and elevated cTn $(\mathrm{HR} 2.05, \mathrm{P}<0.0001)$ were two of the strongest independent associations with mortality. Some individual hospital diagnoses, including haemorrhagic stroke (HR 2.49, $\mathrm{P}<0.0001)$ and sepsis $(2.37, \mathrm{P}<0.0001)$, were associated with mortality. The risk of death associated with elevated cTn diverged early and persisted out to maximum of 3 years of follow-up ( $\log$ rank $\mathrm{P}<0.0001$ ) (figure 1).

\section{PREDICTORS OF CATHETERISATION AND CONSULTATION}

Overall, 3672 (13.8\%) patients had a cardiology consultation during their hospitalisation and those with elevated cTn were more likely to have a consult $(29.6 \%$ vs $10.2 \%$, $\mathrm{P}<0.0001)$. Cardiac catheterisation was performed on 1800 subjects $(6.8 \%$ overall) and was more likely in those with elevated cTn $(20.8 \%$ vs $3.5 \%, \mathrm{P}<0.0001)$. In our logistic regression models, we observed that elevated cTn was the variable with the strongest association to consultation (OR 3.44, $\mathrm{P}<0.0001)$ and to catheterisation (OR 8.16, $\mathrm{P}<0.0001$ ) (table 4 ). In both models, men were more likely to have a consult $(\mathrm{OR} 1.29, \mathrm{P}<0.0001)$ or catheterisation (OR 1.45, $\mathrm{P}<0.0001)$, while African-American subjects were less likely to have either (OR for 
Table 2 Procedures performed and diagnoses made during hospitalisation

\begin{tabular}{|c|c|c|c|c|c|c|c|}
\hline \multirow{2}{*}{ Procedures } & \multicolumn{2}{|c|}{$\begin{array}{l}\text { Troponin }<0.03 \\
\mathrm{n}=21701\end{array}$} & \multicolumn{2}{|c|}{$\begin{array}{l}\text { Troponin } \geq 0.03 \\
\mathrm{n}=4962\end{array}$} & \multirow[t]{2}{*}{$\mathbf{P}$} & \multirow[t]{2}{*}{ OR } & \multirow[t]{2}{*}{$95 \% \mathrm{Cl}$} \\
\hline & & & & & & & \\
\hline Cardiology consult & 2203 & $10.2 \%$ & 1469 & $29.6 \%$ & $<0.0001$ & 3.72 & 3.45 to 4.01 \\
\hline Cardiac MRI & 60 & $0.3 \%$ & 36 & $0.7 \%$ & $<0.0001$ & 2.64 & 1.74 to 3.99 \\
\hline Coronary CT angiography & 363 & $1.7 \%$ & 30 & $0.6 \%$ & $<0.0001$ & 0.36 & 0.25 to 0.52 \\
\hline Chest CT (non-coronary) & 5304 & $24.4 \%$ & 1472 & $29.7 \%$ & $<0.0001$ & 1.30 & 1.22 to 1.40 \\
\hline Single photon emission CT & 2169 & $10.0 \%$ & 324 & $6.5 \%$ & $<0.0001$ & 0.63 & 0.56 to 0.71 \\
\hline Ventilation/perfusion scan & 174 & $0.8 \%$ & 67 & $1.4 \%$ & 0.0002 & 1.69 & 1.28 to 2.25 \\
\hline Cardiopulmonary resuscitation & 48 & $0.2 \%$ & 95 & $1.9 \%$ & $<0.0001$ & 8.81 & 6.21 to 12.48 \\
\hline Cardioversion & 135 & $0.6 \%$ & 74 & $1.5 \%$ & $<0.0001$ & 2.42 & 1.82 to 3.22 \\
\hline Exercise treadmill testing & 2341 & $10.8 \%$ & 324 & $6.5 \%$ & $<0.0001$ & 0.58 & 0.51 to 0.65 \\
\hline Transthoracic echocardiogram & 11421 & $52.6 \%$ & 3938 & $79.4 \%$ & $<0.0001$ & 3.46 & 3.22 to 3.73 \\
\hline Transoesophageal echocardiogram & 463 & $2.1 \%$ & 342 & $6.9 \%$ & $<0.0001$ & 3.40 & 2.94 to 3.92 \\
\hline Coronary catheterisation & 769 & $3.5 \%$ & 1031 & $20.8 \%$ & $<0.0001$ & 7.14 & 6.46 to 7.88 \\
\hline Percutaneous coronary intervention & 122 & $0.6 \%$ & 478 & $9.6 \%$ & $<0.0001$ & 18.86 & 15.42 to 23.06 \\
\hline Coronary bypass & 44 & $0.2 \%$ & 118 & $2.4 \%$ & $<0.0001$ & 11.99 & 8.47 to 16.97 \\
\hline \multicolumn{8}{|l|}{ Hospital diagnoses } \\
\hline Acute myocardial infarction & 55 & $0.3 \%$ & 993 & $20.0 \%$ & $<0.0001$ & 98.47 & 74.90 to 129.45 \\
\hline Chronic cardiovascular disease & 5145 & $23.7 \%$ & 2374 & $47.8 \%$ & $<0.0001$ & 2.95 & 2.77 to 3.15 \\
\hline Arrhythmia* & 3484 & $16.1 \%$ & 1530 & $30.8 \%$ & $<0.0001$ & 2.33 & 2.17 to 2.50 \\
\hline Acute/chronic heart failure & 3305 & $15.2 \%$ & 1936 & $39.0 \%$ & $<0.0001$ & 3.56 & 3.33 to 3.81 \\
\hline Takotsubo cardiomyopathy & 27 & $0.1 \%$ & 101 & $2.0 \%$ & $<0.0001$ & 16.68 & 10.90 to 25.53 \\
\hline Sepsis & 1905 & $8.8 \%$ & 1132 & $22.8 \%$ & $<0.0001$ & 3.07 & 2.83 to 3.33 \\
\hline Chronic kidney disease & 2270 & $10.5 \%$ & 1771 & $35.7 \%$ & $<0.0001$ & 4.75 & 4.42 to 5.11 \\
\hline Acute kidney injury & 2962 & $13.7 \%$ & 1729 & $34.8 \%$ & $<0.0001$ & 3.38 & 3.15 to 3.63 \\
\hline Stroke, haemorrhagic & 618 & $2.9 \%$ & 180 & $3.6 \%$ & 0.004 & 1.28 & 1.08 to 1.52 \\
\hline Stroke, thrombotic & 1700 & $7.8 \%$ & 446 & $9.0 \%$ & 0.007 & 1.16 & 1.04 to 1.30 \\
\hline Aortic dissection & 125 & $0.6 \%$ & 35 & $0.7 \%$ & 0.29 & 1.23 & 0.84 to 1.79 \\
\hline Acute pulmonary embolus & 477 & $2.2 \%$ & 168 & $3.4 \%$ & $<0.0001$ & 1.56 & 1.30 to 1.86 \\
\hline
\end{tabular}

*Atrial or ventricular arrhythmia.

consultation $0.85, \mathrm{P}=0.0006$; OR for catheterisation 0.72 , $\mathrm{P}<0.0001)$.

\section{DISCUSSION}

In this large, single centre cohort study examining the real-world use of cTn, we made several novel and confirmatory observations. Some of these observations suggest patterns of care that appear discordant with best clinical practices. While appropriate use criteria have established standards for use for many cardiovascular tests, the terms overuse and underuse remain difficult to apply. These terms may be relevant when our data are considered in the context of clinical guidelines and other data sources.

Some have suggested that the contemporary application of cardiac biomarker testing for ischaemia is an example of testing overuse. ${ }^{3}$ In our sample of 26663 subjects with cTn testing performed, the proportion with elevation and the positive predictive value of cTn was low. Furthermore, during our study period there were 67829 inpatient admissions. This corresponds to a total of $39.3 \%$ of all inpatients having their cTn measured at some point during their stay. Because of the pattern of pervasive cTn use, which results in a low positive predictive value for diagnosing MI, more judicious use of cTn has been encouraged in editorials and has been the subject of quality improvement initiatives seeking to reduce waste. $^{78}$

The data on ECG use in our cohort raise multiple points for discussion. First, the discrepancy in documentation of ECG between the administrative and manual chart reviews is substantial. As we noted, this is often due to clinically available data (eg, prehospital ECG) not captured because our facility did not have the necessary billing infrastructure. For higher cost testing, such 


\begin{tabular}{|c|c|c|}
\hline & HR & $\mathbf{P}$ \\
\hline Elevated troponin & 2.05 & $<0.0001$ \\
\hline African-American race & 0.86 & $<0.0001$ \\
\hline Male & 1.14 & $<0.0001$ \\
\hline Hypertension* & 0.92 & 0.03 \\
\hline Congestive heart failure* & 1.27 & 0.0004 \\
\hline Stroke or transient ischaemic attack* & 1.16 & 0.009 \\
\hline Chronic obstructive pulmonary disease* & 1.21 & $<0.0001$ \\
\hline Age (per year) & 1.02 & $<0.0001$ \\
\hline Elevated Charlson Comorbidity Index (>4) & 3.33 & $<0.0001$ \\
\hline Aortic dissection† & 1.83 & 0.0002 \\
\hline Acute kidney injury† & 1.31 & $<0.0001$ \\
\hline Arrhythmia† & 1.29 & $<0.0001$ \\
\hline Chronic cardiovascular disease $†$ & 0.85 & $<0.0001$ \\
\hline Stroke, haemorrhagic $†$ & 2.49 & $<0.0001$ \\
\hline Pulmonary embolust & 1.35 & $<0.0001$ \\
\hline Sepsis† & 2.37 & $<0.0001$ \\
\hline
\end{tabular}

*Diagnosis present at time of admission.

†Diagnosis made during hospitalisation.

as echocardiography and nuclear stress testing, we did not observe any discrepancy. Even after manual review, however, some $5 \%$ of patients evaluated with $\mathrm{cTn}$ did not have an ECG performed. Given the simplicity and ubiquity of ECG in the hospital setting, it is perplexing why patients evaluated with $\mathrm{cTn}$, presumably to diagnose myocardial damage, would not undergo an ECG as well.

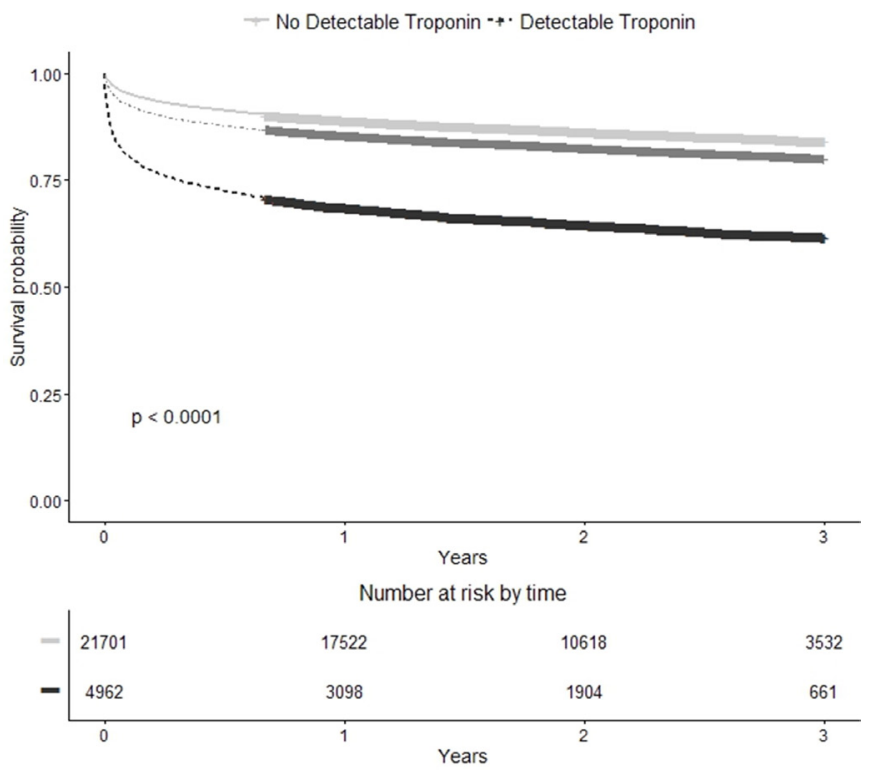

Figure 1 Kaplan-Meier curve for mortality. The light grey line represents the risk of mortality for patients without elevation in cardiac troponin and the dark grey line represents risk for those with elevation in troponin. The medium grey line in between is the risk for the overall population.
Guidelines give a class I recommendation for performing an ECG promptly in patients for whom acute coronary syndrome is suspected. This has a ' $\mathrm{C}$ ' level of evidence, as there does not seem to be any reason why this recommendation should be empirically tested. The message worth communicating is that patients being evaluated for myocardial ischaemia should be evaluated clinically and augmented with both biomarkers and ECG.

The use of cardiology consultation differed nearly threefold between our cohorts. Unlike non-invasive testing, use of expert consultation is much more nuanced and does not lend itself to categorisation schema such as appropriate use criteria. Thus, we cannot comment as to whether consultation was overused or underused. Some patients with elevated cTn and without cardiology consult may have benefited from cardiology care; we cannot ascertain this from our dataset. We observed greater use of echocardiography with elevated cTn, which is reasonably expected. We saw less use of cardiac CT and SPECT with elevated cTn, which is also expected as patients without elevated cTn would be more likely to undergo a non-invasive ischaemia evaluation. These data would be stronger if we had been able to extract information on appropriateness of testing. ${ }^{9}$

A key question raised by our observations is: What is the likely impact of hscTn assays on demand for cardiovascular services? ${ }^{10}$ In 2001, a randomised trial compared the evaluation of emergency department patients with cTn with the then-standard-of-care, Creatine kinase-MB (CKMB). ${ }^{11}$ While subgroup analyses showed significant differences in hospitalisation and hospital costs, these were not different for the cohort as a whole. A more contemporary study from Spain compared conventional and hscTn finding that the newer assay resulted in some improvement of acute triage offset by higher use of invasive procedures and longer hospitalisations. ${ }^{12} \mathrm{~A}$ large registry study from Sweden compared use of cardiovascular services after adoption of a hscTn assay finding modest increases in demand for echocardiography and catheterisation, which did not persist after multivariate regression. ${ }^{13}$ Other studies evaluating adoption of hscTn found no difference in cardiovascular service demand. ${ }^{1415}$ We could only speculate as to how demand for cardiovascular services will change with the transition to hscTn; however, our data on prevalence are informative for future study of this transition. As technology continues to advance rapidly, the medical community and industry partners should consider not just the accuracy of a new test, but also whether the procedure improves outcomes for patients. ${ }^{16}$

Our investigation adds to the body of literature documenting that elevation in cTn is clinically a poor prognostic indicator associated with mortality in both cardiac and non-cardiac conditions. ${ }^{17-19}$ While this mortality risk appears to differ based on the aetiology of the elevation, ${ }^{120}$ our data did not allow for this degree of detailed investigation. We did observe a decreased risk of mortality for African-American patients. A review of literature on 


\begin{tabular}{llcl}
\hline Table 4 Logistic regression models of factors ${ }^{*}$ associated & with cardiology consultation and cardiac catheterisation \\
\hline Cardiology consultation & OR & $\mathbf{9 5 \%} \mathbf{C l}$ & $\mathbf{P}$ \\
\hline Elevated troponin & 3.44 & 3.19 to 3.72 & $<0.0001$ \\
\hline African-American race & 0.85 & 0.77 to 0.93 & 0.0006 \\
\hline Male & 1.29 & 1.20 to 1.39 & $<0.0001$ \\
\hline Congestive heart failure & 1.99 & 1.67 to 2.36 & $<0.0001$ \\
\hline Atrial fibrillation/flutter & 2.10 & 1.84 to 2.40 & $<0.0001$ \\
\hline Chronic obstructive pulmonary disease & 0.82 & 0.69 to 0.97 & 0.0193 \\
\hline Age (per year) & 1.01 & 1.00 to 1.01 & $<0.0001$ \\
\hline Cardiac catheterisation & $\mathbf{O R}$ & $\mathbf{9 5 \%}$ Cl & $\mathbf{P}$ \\
\hline Elevated troponin & 8.16 & 7.35 to 9.06 & $<0.0001$ \\
\hline African-American race & 0.72 & 0.63 to 0.82 & $<0.0001$ \\
\hline Male & 1.45 & 1.31 to 1.61 & $<0.0001$ \\
\hline Chronic kidney disease & 0.39 & 0.29 to 0.53 & $<0.0001$ \\
\hline Coronary artery disease & 2.44 & 2.05 to 2.91 & $<0.0001$ \\
\hline Hypertension & 0.83 & 0.72 to 0.96 & 0.0118 \\
\hline Atrial fibrillation/flutter & 0.66 & 0.51 to 0.86 & 0.0022 \\
\hline Stroke or transient ischaemic attack & 0.41 & 0.31 to 0.54 & $<0.0001$ \\
\hline Chronic obstructive pulmonary disease & 0.59 & 0.44 to 0.78 & 0.0002 \\
\hline Age (per year) & 0.99 & 0.99 to 0.99 & $<0.0001$ \\
\hline Elevated Charlson Comorbidity Ixndex $(>4)$ & 0.44 & 0.35 to 0.54 & $<0.0001$ \\
\hline
\end{tabular}

${ }^{*}$ Medical conditions listed are diagnoses that were present at time of admission.

inpatient mortality and race yields non-uniform findings; some finding African-Americans to have higher mortality, some with lower mortality and some where race was not a relevant factor. ${ }^{21-24}$ Another study evaluating disparities in care and mortality found that women were more likely to receive suboptimal care and that both African-American and female patients have higher AMI mortality than white men. ${ }^{25}$ We observed that use of cardiovascular services was less for women and African-Americans; however, we cannot determine if this represents underuse of services in these groups or overuse in white, male patients. It also remains unclear what impact race and sex will have on hscTn adoption given ongoing disagreement whether hscTn assays should have different cut-offs for different populations. ${ }^{26-28}$

Our study is not without limitations. We were not able to derive a statistical method to determine rise and fall of cTn, as would be preferred for verifying the diagnosis of AMI, within our data set. The data on pre-existing conditions are subject to the limitation of whether they were documented correctly by care providers; data on new hospital diagnoses are drawn from billing and coding. We did not have information on appropriateness of non-invasive imaging or catheterisation. Regression models are an imperfect method for determining risk associations between variables such as sex and ethnicity with outcomes such as consultation and catheterisation. We cannot ascertain any reasons for the disparities observed in women and African-American subjects; these findings should be considered hypothesis generating.

\section{CONCLUSIONS}

Our investigation of the real-world use of cTn demonstrates patterns of cardiovascular service use that may be discordant with best clinical practice. Cardiology consultation is far from universal in evaluating these patients. Elevated cTn continues to be associated with higher mortality, although other factors are stronger predictors of death. Racial and sex-based differences in cardiovascular service use and mortality support continued concern about disparities in care.

Contributors All authors contributed to either the design of the study or collection of the data. All authors participated in the drafting, critical review and final approval of the manuscript. DEW had full access to the data and accepts responsibility for its integrity.

Funding Funding was provided Roche Diagnostics through an investigator-initiated proposal. Roche Diagnostics had no role in the design of the study, the collection and interpretation of the data, or drafting the manuscript.

Competing interests DEW previously served on an advisory board for Roche Diagnostics in 2015. FWP has received grant support from Abbott, Janssen, Roche, ZS Pharma and is a consultant for Beckman, Boehringer-Ingelheim, Cardiorentis, Instrument Labs, Janssen, Phillips, Portola, Prevencio, Singulex, ZS Pharma. The contents of this paper do not represent the views of the US Department of Veterans Affairs or the United States Government.

Ethics approval University of Florida Institutional Review Board.

Provenance and peer review Not commissioned; externally peer reviewed. 
Data sharing statement No additional data are available.

Open Access This is an Open Access article distributed in accordance with the Creative Commons Attribution Non Commercial (CC BY-NC 4.0) license, which permits others to distribute, remix, adapt, build upon this work non-commercially, and license their derivative works on different terms, provided the original work is properly cited and the use is non-commercial. See: http://creativecommons.org/ licenses/by-nc/4.0/

(c) Article author(s) (or their employer(s) unless otherwise stated in the text of the article) 2018. All rights reserved. No commercial use is permitted unless otherwise expressly granted.

\section{REFERENCES}

1. Winchester DE, Burke L, Agarwal N, et al. Predictors of short- and long-term mortality in hospitalized veterans with elevated troponin. J Hosp Med 2016;11:773-7.

2. Thygesen K, Alpert JS, Jaffe AS, et al. Third universal definition of myocardial infarction. J Am Coll Cardiol 2012;60:1581-98.

3. Brush JE, Kaul S, Krumholz HM. Troponin testing for clinicians. J Am Coll Cardiol 2016;68:2365-75.

4. Needham DM, Scales DC, Laupacis A, et al. A systematic review of the Charlson comorbidity index using Canadian administrative databases: a perspective on risk adjustment in critical care research. J Crit Care 2005;20:12-19.

5. Charlson ME, Pompei P, Ales KL, et al. A new method of classifying prognostic comorbidity in longitudinal studies: development and validation. J Chronic Dis 1987;40:373-83.

6. von Elm E, Altman DG, Egger M, et al. The Strengthening the Reporting of Observational Studies in Epidemiology (STROBE) statement: guidelines for reporting observational studies. PLoS Med 2007;4:e296.

7. Larochelle MR, Knight AM, Pantle $\mathrm{H}$, et al. Reducing excess cardiac biomarker testing at an academic medical center. J Gen Intern Med 2014;29:1468-74.

8. CardioPulse articles. Eur Heart J 2016:37:2388-96.

9. Wolk MJ, Bailey SR, Doherty JU, et al. ACCF/AHA/ASE/ASNC/ HFSA/HRS/SCAI/SCCT/SCMR/STS 2013 multimodality appropriate use criteria for the detection and risk assessment of stable ischemic heart disease: a report of the American College of Cardiology Foundation Appropriate Use Criteria Task Force, American Heart Association, American Society of Echocardiography, American Society of Nuclear Cardiology, Heart Failure Society of America, Heart Rhythm Society, Society for Cardiovascular Angiography and Interventions, Society of Cardiovascular Computed Tomography, Society for Cardiovascular Magnetic Resonance, and Society of Thoracic Surgeons. J Am Coll Cardiol 2014;63:380-406.

10. Gale CP, Bueno $\mathrm{H}$. The race for higher sensitivity troponins, but for what prize? Eur Heart J 2016;37:2425-7.

11. Zarich S, Bradley K, Seymour J, et al. Impact of troponin T determinations on hospital resource utilization and costs in the evaluation of patients with suspected myocardial ischemia. Am J Cardiol 2001;88:732-6.
12. Sanchis J, García-Blas S, Mainar L, et al. High-sensitivity versus conventional troponin for management and prognosis assessment of patients with acute chest pain. Heart 2014;100:1591-6.

13. Eggers KM, Lindahl B, Melki D, et al. Consequences of implementing a cardiac troponin assay with improved sensitivity at Swedish coronary care units: an analysis from the SWEDEHEART registry. Eur Heart J 2016;37:2417-24.

14. Chew DP, Zeitz C, Worthley M, et al. Randomized comparison of high-sensitivity troponin reporting in undifferentiated chest pain assessment. Circ Cardiovasc Qual Outcomes 2016;9:542-53.

15. Corsini A, Vagnarelli F, Bugani G, et al. Impact of high-sensitivity Troponin T on hospital admission, resources utilization, and outcomes. Eur Heart J Acute Cardiovasc Care 2015;4:148-57.

16. Ferrante di Ruffano L, Hyde CJ, McCaffery KJ, et al. Assessing the value of diagnostic tests: a framework for designing and evaluating trials. BMJ 2012;344:e686.

17. Yousufuddin $M$, Abdalrhim AD, Wang Z, et al. Cardiac troponin in patients hospitalized with acute decompensated heart failure: a systematic review and meta-analysis. J Hosp Med 2016;11:446-54.

18. Pavasini R, d'Ascenzo F, Campo G, et al. Cardiac troponin elevation predicts all-cause mortality in patients with acute exacerbation of chronic obstructive pulmonary disease: Systematic review and metaanalysis. Int J Cardiol 2015;191:187-93.

19. Michos ED, Wilson LM, Yeh HC, et al. Prognostic value of cardiac troponin in patients with chronic kidney disease without suspected acute coronary syndrome: a systematic review and meta-analysis. Ann Intern Med 2014;161:491-501.

20. Alcalai R, Planer D, Culhaoglu $A$, et al. Acute coronary syndrome vs nonspecific troponin elevation: clinical predictors and survival analysis. Arch Intern Med 2007;167:276-81.

21. Mikhail JN, Nemeth LS, Mueller M, et al. The association of race, socioeconomic status, and insurance on trauma mortality. J Trauma Nurs 2016;23:347-56.

22. Win TT, Davis HT, Laskey WK. Mortality among patients hospitalized with heart failure and diabetes mellitus: results from the national inpatient sample 2000 to 2010. Circ Heart Fail 2016;9:e003023.

23. May FP, Rolston VS, Tapper EB, et al. The impact of race and ethnicity on mortality and healthcare utilization in alcoholic hepatitis: a cross-sectional study. BMC Gastroenterol 2016;16:129.

24. Bime C, Poongkunran C, Borgstrom M, et al. Racial differences in mortality from severe acute respiratory failure in the United States, 2008-2012. Ann Am Thorac Soc 2016;13:2184-9.

25. Li S, Fonarow GC, Mukamal KJ, et al. Sex and race/ethnicity-related disparities in care and outcomes after hospitalization for coronary artery disease among older adults. Circ Cardiovasc Qual Outcomes 2016;9:S36-S44

26. Rubini Giménez M, Twerenbold R, Boeddinghaus J, et al. Clinical effect of sex-specific cutoff values of high-sensitivity cardiac troponin $\mathrm{T}$ in suspected myocardial infarction. JAMA Cardiol 2016;1:912-20.

27. Eggers KM, Jernberg T, Lindahl B. Prognostic importance of sexspecific cardiac troponin T 99(th) percentiles in suspected acute coronary syndrome. Am J Med 2016;129:880.e1-e12.

28. Shah AS, Griffiths M, Lee KK, et al. High sensitivity cardiac troponin and the under-diagnosis of myocardial infarction in women: prospective cohort study. BMJ 2015;350:g7873. 